\title{
Asymptotic Padé approximant predictions: Up to five loops in QCD and SQCD
}

\author{
J. Ellis* \\ Theory Division, CERN, CH-1211 Geneva 23, Switzerland \\ I. Jack ${ }^{\dagger}$ and D. R. T. Jones \\ Department of Mathematical Sciences, University of Liverpool, Liverpool L69 3BX, United Kingdom \\ M. Karliner ${ }^{\S}$ \\ Raymond and Beverly Sackler Faculty of Exact Sciences, School of Physics and Astronomy, \\ Tel-Aviv University, 69978 Tel-Aviv, Israel \\ M. A. Samuel \\ Department of Physics, Oklahoma State University, Stillwater, Oklahoma 74078 \\ and SLAC, Stanford University, Stanford, California 94309 \\ (Received 10 October 1997; published 10 February 1998)
}

\begin{abstract}
We use asymptotic Padé approximants to predict the four- and five-loop $\beta$ functions in QCD and $N=1$ supersymmetric QCD, as well as the quark mass anomalous dimensions in Abelian and non-Abelian gauge theories. We show how the accuracy of our previous $\beta$-function predictions at the four-loop level may be further improved by using estimators weighted over negative numbers of flavors (WAPAP's). The accuracy of the improved four-loop results encourages confidence in the new five-loop $\beta$-function predictions that we present. However, the WAPAP approach does not provide improved results for the anomalous mass dimension, or for Abelian theories. [S0556-2821(98)05105-4]
\end{abstract}

PACS number(s): 11.10.Gh, 11.15.Bt

\section{INTRODUCTION}

One of the greatest challenges in QCD is the calculation of higher orders in perturbation theory. Phenomenologically, these are important because the relatively large value of $\alpha_{s}$ at accessible energies implies that many orders of perturbation theory are required in order to make precise quantitative tests. Theoretically, one expects the coefficients of the perturbative series for many QCD quantities to diverge factorially, and the rates of these divergences may cast light on issues in nonperturbative QCD, such as the existence and magnitudes of condensates and higher-twist effects [1]

On the other hand, while progress in the exact calculations of higher-order terms in perturbative QCD series has been startling, with many new multiloop results having recently become available [2], existing perturbative techniques may not enable much further progress in exact calculations to be made in the near future. Thus various approximate techniques and numerical estimates may have a useful role to play. Among these, one may mention exact calculations of certain perturbative coefficients in the large- $N_{F}$ limit, and the emerging lore of renormalons [1]. Also of potential use in QCD are Padé approximants (PA's), as described in Sec. II of this paper, which have previously demonstrated their utility in applications to problems in condensed-matter physics

\footnotetext{
*Email address: John.Ellis@cern.ch

†Email address: dij@amtp.liv.ac.uk

†Email address: drtj@amtp.liv.ac.uk

${ }^{\S}$ Email address: marek@vm.tau.ac.il

"Deceased.
}

and statistical mechanics [3]. In recent years, these have been applied to obtain successful numerical predictions in various quantum field theories, including QCD, and justifications for some of these successes have been found in some mathematical theorems [4] on the convergence and renormalization-scale invariance of PA's. These theorems apply, in particular, to perturbative QCD series dominated by renormalon singularities, and in the large- $\beta_{0}$ limit.

Based on these theorems, a new method was introduced [5] for estimating the next-order coefficients in perturbative quantum field theory series on the basis of the known lowerorder results and plausible conjectures on the likely highorder behavior of the series, as also reviewed in Sec. II. This method "corrects" the conventional Padé approximant prediction (PAP) of the next term in the series by using an asymptotic error formula, providing improved predictions that we call asymptotic Padé approximant predictions (APAP's).

APAP's have already provided successful predictions for the perturbative coefficients in the subsequent calculation of the four-loop $\beta$ function in QCD, as discussed in Sec. III, and have also provided interesting results in $N=1$ supersymmetric QCD (SQCD) [6]. The purpose of this paper is to provide a more complete account of these predictions, to show how their accuracy may be improved in certain cases by a judicious weighting over negative numbers of flavors $N_{F}$, and to extend these predictions to five loops in QCD in Sec. V, and to SQCD in Sec. VI. We also discuss analogous predictions for the QCD anomalous quark mass dimension in Sec. VII where the "regular" APAP gives very good results, but the new weighting method does not improve matters. In 
Sec. VIII we consider Abelian gauge theories, with less successful results.

Before deriving these predictions, there is a technical issue which should be clarified, that may also illuminate an interesting physics point. As a general rule, $\beta$ functions are scheme-dependent beyond one loop, and a theory with a single perturbative coupling constant $g$, such as QCD, is scheme-dependent beyond two loops, if one considers analytic redefinitions of $g$. In particular, the QCD $\beta$ function can be transformed to zero beyond two loops, by making a suitable choice of renormalization scheme. ${ }^{1}$ In our analysis of QCD, we use the modified minimal subtraction scheme $(\overline{\mathrm{MS}}$ ), and in $N=1 \mathrm{SQCD}$ we favor the dimensional reduction (DRED) scheme. ${ }^{2}$ The successes of the APAP procedure indicate that asymptotia and the convergence of PAP's are remarkably precocious in these schemes. In the SQCD case, there exists an alternative scheme [Novikov-ShifmanVainshtein-Zakharov (NSVZ)] [7], associated with the Wilsonian action, in which there is an all-orders relation between $\beta_{g}$ and the quark anomalous dimension $\gamma_{q}$. The NSVZ scheme differs perturbatively from DRED [8], and therefore provides a distinct test for the APAP method. We compare predictions for $\beta_{g}$ in both DRED and NSVZ, finding that they are less compelling in the latter case: perhaps minimal subtraction schemes are more amenable to Padé techniques? If so, it would be interesting to fathom the reason. As already noted, these techniques are not so successful for the quark mass anomalous dimension, or for Abelian theories. Perhaps these instances also provide clues when and why the Padé magic works.

\section{FORMALISM}

We start by recalling relevant aspects of the formalism for PA's and APAP's, and establishing our notation. For a generic perturbative series

$$
S(x)=\sum_{n=0}^{N_{\max }} S_{n} x^{n}
$$

the Padé approximant $[N / M](x)$ is given by [3]

$$
[N / M]=\frac{a_{0}+a_{1} x+\cdots a_{N} x^{N}}{b_{0}+b_{1} x+\cdots b_{M} x^{M}}
$$

with $b_{0}=1$, and the other coefficients chosen so that

$$
[N / M]=S+O\left(x^{N+M+1}\right) .
$$

The coefficient of the $x^{N+M+1}$ term in Eq. (2.3) is the PAP estimate $S_{N+M+1}^{\mathrm{PAP}}$ of $S_{N+M+1}$. If the perturbative coefficients $S_{n}$ diverge as $n$ ! for large $n$, it is possible to show [4] that the relative error

\footnotetext{
${ }^{1}$ In fact, it can even be transformed to zero beyond one loop by a nonanalytic redefinition of $g$ involving $\ln g$ : such redefinitions are associated with the Wilsonian action in supersymmetric theories.

${ }^{2} \mathrm{We}$ recall that DRED corresponds to minimal subtraction in conjunction with regularization by dimensional reduction.
}

$$
\delta_{N+M+1} \equiv \frac{S_{N+M+1}^{\mathrm{PAP}}-S_{N+M+1}}{S_{N+M+1}}
$$

has the asymptotic form

$$
\delta_{N+M+1} \simeq-\frac{M ! \mathcal{A}^{M}}{L_{[N / M]}^{M}}
$$

as $N \rightarrow \infty$, for fixed $M$, where

$$
L_{[N / M]}=N+M+a M+b,
$$

and $\mathcal{A}, a$, and $b$ are constants. This theorem not only guarantees the convergence of the PAP's, but also specifies the asymptotic form of the corrections.

The idea of APAP's is to fit the magnitude of this asymptotic correction using the known low-order perturbative coefficients, and apply the resulting numerical correction to the naive PAP's. In the applications discussed in this paper, we work with [0/1], [1/1], and [2/1] PA's, so that $M$ $=1$ throughout. For example, four-loop predictions are obtained as follows. In the case $N_{\max }=2$, the [1/1] Padé leads to the naive PAP $S_{3}^{\mathrm{PAP}}=S_{2}^{2} / S_{1}$. The improved APAP estimate is then given by

$$
S_{3}^{\mathrm{APAP}}=\frac{S_{3}^{\mathrm{PAP}}}{1+\delta_{3},},
$$

where, motivated by its appropriateness in $\phi^{4}$ field theory, we choose $a+b=0$ in the QCD application discussed in Sec. III, and $\mathcal{A}$ is then determined by comparing $S_{2}$ to $S_{2}^{\mathrm{PAP}}=S_{1}^{2} / S_{0}$. Alternatively, we could have chosen a value of $\mathcal{A}$ and determined $a+b$ from $\delta_{2}$. However, as we shall see, when we go to five loops, knowledge of $\delta_{2}$ and $\delta_{3}$ enables us to fit both $\mathcal{A}$ and $a+b$ simultaneously.

\section{APPLICATION TO THE FOUR-LOOP $\beta$ FUNCTION IN QCD}

The APAP method was applied in Ref. [5] to estimate the four-loop QCD $\beta$-function coefficient $\beta_{3}$, on the basis of the lower-order terms

$$
\begin{gathered}
\beta_{0}=\frac{11}{3} C_{A}-\frac{4}{3} T_{F} N_{F}, \\
\beta_{1}=\frac{34}{3} C_{A}^{2}-4 C_{F} T_{F} N_{F}-\frac{20}{3} C_{A} T_{F} N_{F}, \\
\beta_{2}=\frac{2857}{54} C_{A}^{3}+N_{F}\left[2 C_{F}^{2} T_{F}-\frac{205}{9} C_{F} C_{A} T_{F}-\frac{1415}{27} C_{A}^{2} T_{F}\right] \\
+N_{F}^{2}\left[\frac{44}{9} C_{F} T_{F}^{2}+\frac{158}{27} C_{A} T_{F}^{2}\right],
\end{gathered}
$$

known before the appearance of the explicit four-loop calculation [9]. The quadratic Casimir coefficients $C_{A}$ and $C_{F}$ for the adjoint and fundamental representations are given for the case of $S U\left(N_{C}\right)$ by

$$
C_{A}=N_{C}, \quad C_{F}=\frac{N_{C}^{2}-1}{2 N_{C}},
$$

and we assume the standard normalisation so that $T_{F}=\frac{1}{2}$. We denote by $N_{A}$ the number of group generators, so that for $S U\left(N_{C}\right)$ we have $N_{A}=N_{C}^{2}-1$. 
TABLE I. Exact four-loop results for the QCD $\beta$ function, compared with the original APAP's in the first column, and improved APAP's obtained from a weighted average over negative $N_{F}$ (WAPAP), as discussed in the text. The numbers in parentheses are the error estimates from Ref. [5].

\begin{tabular}{lccccc}
\hline \hline & APAP & EXACT & $\%$ DIFF & WAPAP & $\%$ DIFF \\
\hline$A_{3}$ & $23,600(900)$ & 24,633 & $-4.20(3.70)$ & 24,606 & -0.11 \\
$B_{3}$ & $-6,400(200)$ & $-6,375$ & $-0.39(3.14)$ & $-6,374$ & -0.02 \\
$C_{3}$ & $350(70)$ & 398.5 & $-12.2(17.6)$ & 402.5 & -1.00 \\
$D_{3}$ & input & 1.499 & - & input & - \\
\hline \hline
\end{tabular}

We recall that $\beta_{3}$ is a polynomial in the number of flavors $N_{F}$,

$$
\beta_{3}=A_{3}+B_{3} N_{F}+C_{3} N_{F}^{2}+D_{3} N_{F}^{3}
$$

where $D_{3}=1.499$ (for $N_{C}=3$ ) was already known from large- $N_{F}$ calculations. To justify applying the estimate (2.5), we assume that the $\beta_{n} \sim n$ ! for large $n$, as discussed in Ref. [5]. The predictions for $A_{3}, B_{3}$, and $C_{3}$ resulting from fitting the APAP results for $0 \leqslant N_{F} \leqslant 4$ to a polynomial of form (2.5) are compared to the exact results in the first columns of Table I.

The exact four-loop coefficient of the QCD $\beta$ function for $N_{C}$ colors is taken from the calculation of Ref. [9], which was published after the APAP estimate,

$$
\begin{aligned}
\beta_{3}= & C_{A}^{4}\left(\frac{150653}{486}-\frac{44}{9} \zeta_{3}\right)+\frac{d_{A}^{a b c d} d_{A}^{a b c d}}{N_{A}}\left(-\frac{80}{9}+\frac{704}{3} \zeta_{3}\right) \\
& +N_{F}\left[C_{A}^{3} T_{F}\left(-\frac{39143}{81}+\frac{136}{3} \zeta_{3}\right)+C_{A}^{2} C_{F} T_{F}\left(\frac{7073}{243}-\frac{656}{9} \zeta_{3}\right)\right. \\
& +C_{A} C_{F}^{2} T_{F}\left(-\frac{4204}{27}+\frac{352}{9} \zeta_{3}\right)+46 C_{F}^{3} T_{F} \\
& \left.+\frac{d_{F}^{a b c d} d_{A}^{a b c d}}{N_{A}}\left(\frac{512}{9}-\frac{1664}{3} \zeta_{3}\right)\right]+N_{F}^{2}\left[C_{A}^{2} T_{F}^{2}\left(\frac{7930}{81}+\frac{224}{9} \zeta_{3}\right)\right. \\
& +C_{F}^{2} T_{F}^{2}\left(\frac{1352}{27}-\frac{704}{9} \zeta_{3}\right)+C_{A} C_{F} T_{F}^{2}\left(\frac{17152}{243}+\frac{448}{9} \zeta_{3}\right) \\
& \left.+\frac{d_{F}^{a b c d} d_{F}^{a b c d}}{N_{A}}\left(-\frac{704}{9}+\frac{512}{3} \zeta_{3}\right)\right]+N_{F}^{3}\left[\frac{424}{243} C_{A} T_{F}^{3}\right. \\
& \left.+\frac{1232}{243} C_{F} T_{F}^{3}\right],
\end{aligned}
$$

where $\zeta_{3} \equiv \zeta(3)=1.2020569 \ldots$. The quartic Casimir coefficients in Eq. (3.4) are given for $S U\left(N_{C}\right)$ by

$$
\begin{gathered}
d_{A}^{a b c d} d_{A}^{a b c d}=\frac{N_{C}^{2}\left(N_{C}^{2}-1\right)\left(N_{C}^{2}+36\right)}{24}, \\
d_{F}^{a b c d} d_{A}^{a b c d}=\frac{N_{C}\left(N_{C}^{2}-1\right)\left(N_{C}^{2}+6\right)}{48}, \\
d_{F}^{a b c d} d_{F}^{a b c d}=\frac{\left(N_{C}^{2}-1\right)\left(N_{C}^{4}-6 N_{C}^{2}+18\right)}{96 N_{C}^{2}} .
\end{gathered}
$$

For $N_{C}=3$, one obtains

$$
\beta_{3} \approx 29243.0-6946.30 N_{F}+405.089 N_{F}^{2}+1.49931 N_{F}^{3} \text {, }
$$

whereas $\beta_{3}$ is given by the coefficients shown in Table I when one omits the quartic Casimir contributions.

These quartic Casimir terms appear for the first time at four-loop order. They are analogous to the light-by-light scattering terms in $(g-2)_{\mu}$, and PA-based techniques cannot estimate them on the basis of lower-order terms with different group-theoretical factors. Such terms are known to be important in $(g-2)_{\mu}$, but were relatively unimportant in previous perturbative QCD applications. In the case of $\beta_{3}$, they turn out to be about $15-20 \%$ for small $N_{F}$, but are non-negligible for $N_{F} \sim 5$. Setting these terms aside, the agreement between the predictions of Ref. [5] and the exact results of Ref. [9] is remarkable. The predictions we present in the rest of this paper should all be understood as applying to perturbative coefficients without the higher-order analogs of such quartic Casimir terms.

Following Ref. [5], the same APAP method was applied in [6] to estimate the four-loop $\beta$ function in SQCD. The agreement with known results was again encouraging, and the APAP provided a prediction $\alpha \approx 2.4$ for the unknown constant [8] in the four-loop SQCD $\beta$ function, as also discussed in Sec. V.

\section{WEIGHTED APAP'S IN QCD}

Before going on to make new predictions for QCD and SQCD at the five-loop level, we first draw attention to a refinement that offers an improvement on APAP's in the four-loop QCD case. As can be seen in Table I, the signs of the coefficients $A_{3}, B_{3}$, and $C_{3}$ alternate. A corollary of this is that the APAP predictions for $N_{F} \sim 5$ are sensitive to cancellations, and relatively inaccurate. Moreover, $S_{3}^{\mathrm{APAP}}$ has a pole at $N_{F}=8.05$ because $\beta_{1}$ vanishes there. Conversely, the numerical analysis is relatively stable for (fictitious) $N_{F}<0$ - there are no poles at negative $N_{F}$ and $S_{3}^{\mathrm{APAP}}$ is quite smooth at $N_{F}=0$, thanks to the pure gluon contribution. We have observed empirically that more accurate predictions for the coefficients $A_{3}, B_{3}$, and $C_{3}$ are obtained if one makes polynomial fits for some range of negative values of $N_{F}$. This does not of course imply the existence of a physical theory for negative $N_{F}$. At any finite order, the Padé approximant prediction is trivially an analytic function of $N_{F}$ (except for isolated poles), and our goal is simply to find the best match to a polynomial. Is there some systematic procedure that we can adopt to determine the appropriate range of $N_{F}$ to use in the fit? The following is one method we have explored.

We choose a range $-N_{F}^{\max } \leqslant N_{F} \leqslant 0$ over which we fit values of $\mathcal{A}$ using the APAP formulas of Sec. III, and we determine the arithmetic mean of the corresponding values of $\mathcal{A}$. We use this mean value of $\mathcal{A}$ to estimate $\beta_{3}$ for each of the chosen values of $N_{F}$, and fit to the polynomial form (3.3). We hypothesize that the most accurate results for the coefficients $A_{3}, B_{3}$, and $C_{3}$ may be obtained when they contribute with equal weights to the fit: certainly, one cannot expect that any coefficient that has a small weight in the fit will be estimated reliably. For a given $N_{F}^{\max }$, the overall weights in the fit are $A_{3}, B_{3} N_{F}^{\max } / 2$, and 
TABLE II. Comparison of WAPAP and exact results for the exact four-loop $\beta$ function in QCD (omitting quartic Casimir terms), for various values of $N_{C}$.

\begin{tabular}{|c|c|c|c|}
\hline & WAPAP & exact & $\%$ error \\
\hline \multicolumn{4}{|c|}{$N_{C}=2$} \\
\hline$A_{3}$ & $4.88 \times 10^{3}$ & 4866 & 0.42 \\
\hline$B_{3}$ & $-1.86 \times 10^{3}$ & -1854 & 0.48 \\
\hline$C_{3}$ & 174 & 170.5 & 2.0 \\
\hline \multicolumn{4}{|c|}{$N_{C}=3$} \\
\hline$A_{3}$ & $2.467 \times 10^{4}$ & 24633 & 0.13 \\
\hline$B_{3}$ & $-6.383 \times 10^{3}$ & -6375 & 0.13 \\
\hline$C_{3}$ & 405 & 398.5 & 1.6 \\
\hline \multicolumn{4}{|c|}{$N_{C}=4$} \\
\hline$A_{3}$ & $7.790 \times 10^{4}$ & 77852 & 0.06 \\
\hline$B_{3}$ & $-1.521 \times 10^{4}$ & -15210 & 0.03 \\
\hline$C_{3}$ & 729 & 717.2 & 1.6 \\
\hline \multicolumn{4}{|c|}{$N_{C}=5$} \\
\hline$A_{3}$ & $1.901 \times 10^{5}$ & 190068 & 0.04 \\
\hline$B_{3}$ & $-2.976 \times 10^{4}$ & -29800 & -0.12 \\
\hline$C_{3}$ & $1.14 \times 10^{3}$ & 1127 & 1.6 \\
\hline \multicolumn{4}{|c|}{$N_{C}=6$} \\
\hline$A_{3}$ & $3.943 \times 10^{5}$ & 394,125 & 0.03 \\
\hline$B_{3}$ & $-5.149 \times 10^{4}$ & $-51,580$ & -0.17 \\
\hline$C_{3}$ & $1.65 \times 10^{3}$ & $1,627.5$ & 1.6 \\
\hline \multicolumn{4}{|c|}{$N_{C}=10$} \\
\hline$A_{3}$ & $3.043 \times 10^{6}$ & $3,041,089$ & 0.05 \\
\hline$B_{3}$ & $-2.388 \times 10^{5}$ & $-239,384$ & -0.25 \\
\hline$C_{3}$ & $4.62 \times 10^{3}$ & 4,540 & 1.7 \\
\hline
\end{tabular}

$C_{3} N_{F}^{\max }\left(2 N_{F}^{\max }+1\right) / 6$. We then estimate $B_{3}$ as follows. We take the two values of $B_{3}$ corresponding to the values of $N_{F}^{\max }$ for which the $A_{3}$ and $B_{3}$ weights are most nearly equal. Let us call these values of $B_{3}, B_{3}^{(1)}$, and $B_{3}^{(2)}$, and the corresponding weights $B_{3}^{W(1)}$ and $B_{3}^{W(2)}$. Our prediction for $B_{3}$ is then

$$
B_{3}=\frac{\Delta_{2} B_{3}^{(1)}+\Delta_{1} B_{3}^{(2)}}{\Delta_{1}+\Delta_{2}},
$$

where $\Delta_{1,2}=\left|B_{3}^{W(1,2)}-A_{3}^{W(1,2)}\right|$. We estimate $C_{3}$ in a similar fashion. Both the $B_{3}$ and $C_{3}$ calculations yield a result for $A_{3}$, obtained as in Eq. (4.1): we take the mean of these two values as our prediction for $A_{3}$.

Table I, in the column labeled WAPAP, shows the results we obtain using this procedure. We see that the latter are significantly more accurate than the ones obtained using the APAP's for $0 \leqslant N_{F} \leqslant 4$. The values of $N_{F}^{\max }$ selected by WAPAP are 7 and 8 for $B_{3}$, and 13 and 14 for $C_{3}$.

Table II compares the WAPAP predictions obtained in this way with the known exact results (omitting quartic Casimir contributions) in QCD for various values of $N_{C}$. The agreement is certainly impressive, even compared with the APAP results shown in Table I. Since the numerical value of the coefficient $C_{3}$ is relatively small, corresponding (in the

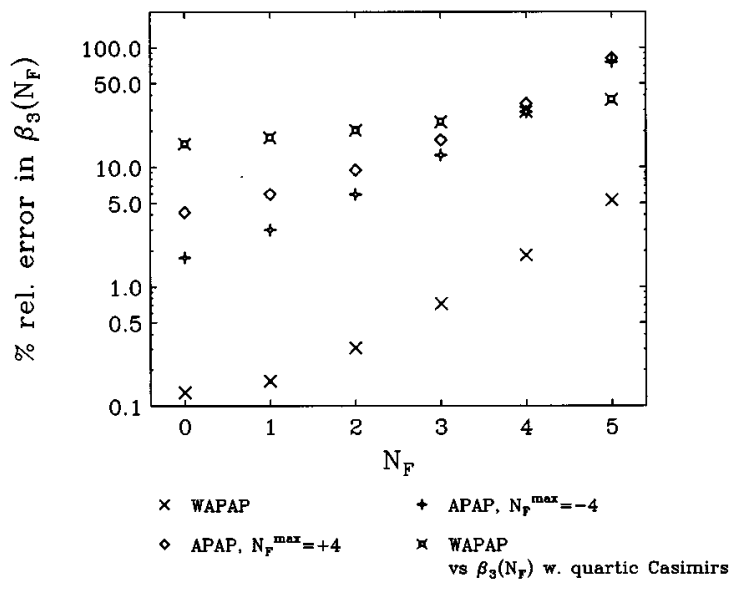

FIG. 1. Predictions for $\beta_{3}$, as function of $N_{F}$, for $N_{C}=3$. The percentage relative errors are obtained using various APAP-based estimation schemes: naive APAP's fitted with positive $N_{F} \leqslant 4$ (diamonds), naive APAP's fitted with negative $N_{F} \geqslant-4$, WAPAP's compared to the exact value of $\beta_{3}$ including quartic Casimir terms, and WAPAP's compared to $\beta_{3}$ without quartic Casimir terms (crosses).

case $N_{C}=3$ ) to the relatively large value $N_{F}^{\max }=14$ mentioned above, it is perhaps not surprising that the percentage error in the estimate of this coefficient is larger than for either $A_{3}$ or $B_{3}$.

Figure 1 graphically displays our resulting predictions for $\beta_{3}$, as a function of $N_{F}$ for the most interesting case $N_{C}$ $=3$. We plot the percentage relative errors obtained using various APAP-based estimation schemes: naive APAP's fitted with positive $N_{F} \leqslant 4$ (diamonds), naive APAP's fitted with negative $N_{F} \geqslant-4$, WAPAP's compared to the exact value of $\beta_{3}$ including quartic Casimir terms, and WAPAP's compared to $\beta_{3}$ without quartic Casimir terms (crosses). We see that the latter are the most accurate for $\beta_{3}$ in QCD. In Fig. 2 we show the error in the WAPAP prediction for $\beta_{3}$ as a function of $N_{F}$, and for $N_{C}=3,4,5,6,7$, and 10, once again omitting quartic Casimir terms from the exact result. The accuracy of these predictions is our best evidence for believing in the utility of the WAPAP method.

To anticipate the obvious question: we have explored whether this WAPAP procedure gives significantly better results than the conventional APAP's for the other perturbative series considered in this paper, namely, the SQCD $\beta$ function and the anomalous dimension of the quark mass. As we discuss in Secs. VII and VIII, the remarkable success of the method at four loops is not repeated for other cases, but there is distinct evidence (provided by large- $N_{F}$-expansion results) that WAPAP leads to more reliable predictions at five loops. However, we feel that the results in Tables I and II already provide ample motivation for the QCD WAPAP calculation of $\beta_{4}$ described in Sec. V.

\section{FIVE-LOOP PREDICTIONS IN QCD}

We now outline the application of the APAP method to estimate the five-loop $\beta$ function coefficients $\beta_{4}$ in QCD, using our knowledge of the corresponding $\beta_{0}$ to $\beta_{3}$. The standard $[2,1]$ Padé leads to the estimate 


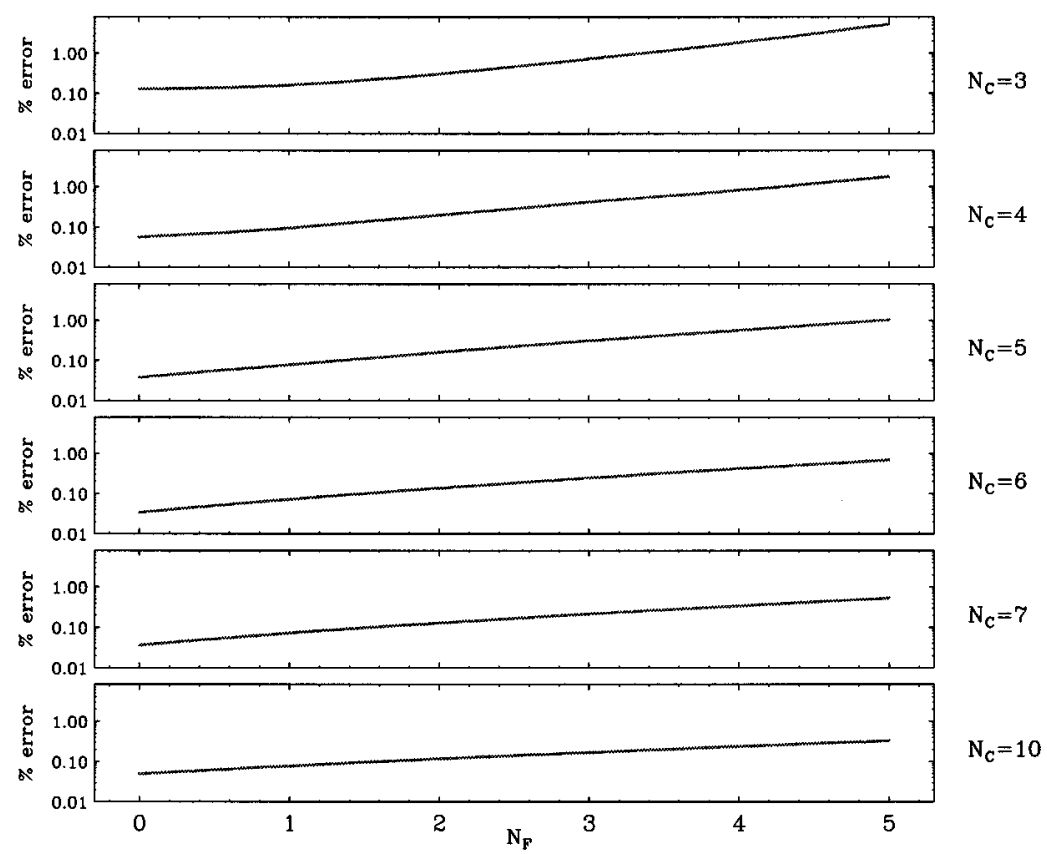

FIG. 2. The percentage relative errors in the WAPAP prediction for $\beta_{3}$ (compared to the exact result with quartic Casimir terms omitted), plotted vs $N_{F}$ for $N_{C}=3,4,5,6,7$, and 10 .

$$
\beta_{4}^{\mathrm{PAP}}=\frac{\beta_{3}^{2}}{\beta_{2}} .
$$

This is then corrected in a similar fashion to Eq. (2.7),

$$
\beta_{4}^{\mathrm{APAP}}=\frac{\beta_{4}^{\mathrm{PAP}}}{1+\delta_{4}}
$$

where, according to Eqs. (2.5) and (2.6), $\delta_{4}$ is given asymptotically by

$$
\delta_{4}=-\frac{\mathcal{A}}{L_{[2 / 1]}}=-\frac{\mathcal{A}}{3+a+b} .
$$

To estimate $\delta_{4}$, we therefore need to know both $\mathcal{A}$ and $a+b$. These can be deduced from the lower-order relative errors $\delta_{2}$ and $\delta_{3}$, as defined in Eq. (2.4), for which we use the asymptotic estimates (2.5):

$$
\frac{\mathcal{A}}{\delta_{2}}=-(1+a+b), \quad \frac{\mathcal{A}}{\delta_{3}}=-(2+a+b),
$$

from which we obtain ${ }^{3} \mathcal{A}$ and $a+b$.

We now calculate the WAPAP for the five-loop QCD $\beta$ function, which we parametrize as

$$
\beta_{4}=A_{4}+B_{4} N_{F}+C_{4} N_{F}^{2}+D_{4} N_{F}^{3}+E_{4} N_{F}^{4} \text {. }
$$

Once again we can input the coefficient of the highest power in $N_{F}$, which is given in this case by [10]

$$
E_{4}=-4 T_{F}^{4}\left[(288 \zeta(3)+214) C_{F}+(480 \zeta(3)-229) C_{A}\right] / 243,
$$

\footnotetext{
${ }^{3}$ The fitted value of $a+b$ is not necessarily close to the value zero assumed in the estimate of $\beta_{3}$ in QCD.
}

TABLE III. WAPAP's for the five-loop QCD $\beta$ function, calculated both with (with $Q$ ) and without (without $Q$ ) the four-loop quartic Casimir terms in $\beta_{3}$. The values of $N_{F}^{\max }$ used range between 5 and 117 in the with $Q$ case, and between 4 and 108 in the without $Q$ case, being largest for large $N_{C}$ and for $D_{4}$.

\begin{tabular}{lcccrr}
\hline \hline$N_{C}$ & 2 & 3 & \multicolumn{1}{c}{4} & \multicolumn{1}{c}{5} & \multicolumn{1}{c}{10} \\
\hline$A_{4}($ with $Q)$ & $1.48 \times 10^{5}$ & $7.59 \times 10^{5}$ & $2.77 \times 10^{6}$ & $7.92 \times 10^{6}$ & $2.31 \times 10^{8}$ \\
$A_{4}($ without $Q)$ & $6.41 \times 10^{4}$ & $4.88 \times 10^{5}$ & $2.06 \times 10^{6}$ & $6.28 \times 10^{6}$ & $2.01 \times 10^{8}$ \\
$B_{4}($ with $Q)$ & $-5.51 \times 10^{4}$ & $-2.19 \times 10^{5}$ & $-6.39 \times 10^{5}$ & $-1.50 \times 10^{6}$ & $-2.28 \times 10^{7}$ \\
$B_{4}($ without $Q)$ & $-3.04 \times 10^{4}$ & $-1.56 \times 10^{5}$ & $-4.97 \times 10^{5}$ & $-1.22 \times 10^{6}$ & $-1.95 \times 10^{7}$ \\
$C_{4}($ with $Q)$ & $6.96 \times 10^{3}$ & $2.05 \times 10^{4}$ & $4.68 \times 10^{4}$ & $9.00 \times 10^{4}$ & $7.07 \times 10^{5}$ \\
$C_{4}($ without $Q)$ & $4.69 \times 10^{3}$ & $1.64 \times 10^{4}$ & $3.93 \times 10^{4}$ & $7.72 \times 10^{4}$ & $6.23 \times 10^{5}$ \\
$D_{4}($ with $Q)$ & -21.8 & -49.8 & -89.8 & -142 & -575 \\
$D_{4}($ without $Q)$ & -28.3 & -60.5 & -105 & -163 & -640 \\
$E_{4}$ (input) & -1.15 & -1.84 & -2.51 & -3.17 & -6.43 \\
\hline \hline
\end{tabular}


TABLE IV. WAPAP's for the five-loop QCD $\beta$ function, calculated with and without the four-loop quartic Casimir terms, but without inputting the known exact values of $E_{4}$. It is encouraging to compare the output values with the last row in Table III. The values of $N_{F}^{\max }$ used range between 5 and 81 in the with $Q$ case, and between 4 and 104 in the without $Q$ case.

\begin{tabular}{lcccrc}
\hline \hline$N_{C}$ & 2 & 3 & \multicolumn{1}{c}{4} & \multicolumn{1}{c}{5} & \multicolumn{1}{c}{10} \\
\hline$A_{4}($ with $Q)$ & $1.45 \times 10^{5}$ & $7.51 \times 10^{5}$ & $2.75 \times 10^{6}$ & $7.87 \times 10^{6}$ & $2.30 \times 10^{8}$ \\
$A_{4}($ without $Q)$ & $6.38 \times 10^{4}$ & $4.85 \times 10^{5}$ & $2.05 \times 10^{6}$ & $6.24 \times 10^{6}$ & $2.00 \times 10^{8}$ \\
$B_{4}($ with $Q)$ & $-5.53 \times 10^{4}$ & $-2.20 \times 10^{5}$ & $-6.41 \times 10^{5}$ & $-1.51 \times 10^{6}$ & $-2.29 \times 10^{7}$ \\
$B_{4}($ without $Q)$ & $-3.05 \times 10^{4}$ & $-1.57 \times 10^{5}$ & $-4.99 \times 10^{5}$ & $-1.22 \times 10^{6}$ & $-1.96 \times 10^{7}$ \\
$C_{4}($ with $Q)$ & $6.72 \times 10^{3}$ & $1.97 \times 10^{4}$ & $4.50 \times 10^{4}$ & $8.66 \times 10^{4}$ & $6.81 \times 10^{5}$ \\
$C_{4}($ without $Q)$ & $4.52 \times 10^{3}$ & $1.58 \times 10^{4}$ & $3.79 \times 10^{4}$ & $7.43 \times 10^{4}$ & $5.99 \times 10^{5}$ \\
$D_{4}($ with $Q)$ & -28.3 & -93.8 & -226 & -389 & $-1,730$ \\
$D_{4}($ without $Q)$ & -72.7 & -163 & -287 & -446 & $-1,750$ \\
$E_{4}($ with $Q)$ & -0.974 & -2.03 & -3.07 & -4.06 & -8.73 \\
$E_{4}($ without $Q)$ & -1.61 & -2.56 & -3.45 & -4.33 & -8.64 \\
\hline \hline
\end{tabular}

using which we obtain the five-loop results shown in Table III.

Notice that in Table III we include results corresponding to both the inclusion (with $Q$ ) and the omission (without $Q$ ) of the quartic Casimir contributions to the four-loop coefficients, obtained from Eq. (3.4). The former (latter) results should of course be compared with contributions including (excluding) such terms at five loops when (and if) such results become available. Of course, at five-loop order we may expect to encounter new higher-order Casimir terms, which should in any event be omitted in the comparison. We can only hope that such contributions are relatively unimportant, which is the case for the quartic terms in $\beta_{3}$ for small $N_{F}$. We anticipate that the percentage errors of the without $Q$ estimates of the nonquartic terms in the coefficients are likely to be the smallest, whereas the best estimate of the full coefficients may be provided by the with $Q$ estimates.

We show in Table IV the results obtained if we choose not to input the value of $E_{4}$, but rather predict that as well. As can be seen, the results for $A_{4}, B_{4}$, and $C_{4}$, in particular, are very stable. Moreover, the prediction for $E_{4}$ is encouragingly close to the true value, considering the extreme smallness of $E_{4}$ compared to $A_{4}$.

It is not possible to state precise errors for the type of prediction discussed in this paper. In Ref. [5] we gave certain estimates of the uncertainties, which turned out to be in the right ballpark if quartic Casimir terms are omitted in the comparison, as reported in Table I. The appearance of such new quartic terms is characteristic of the type of theoretical "systematic error" that cannot be foreseen. In the case of our $\beta_{4}$ predictions in QCD, we draw the reader's attention to the differences between the with $Q$ and without $Q$ entries in Table III, and to the differences between these and the corresponding entries in Table IV, obtained without using the known values of $E_{4}$ as inputs. The most accurate estimates of the full coefficients are likely to be the with $Q$ entries in Table III, but the uncertainties are unlikely to be smaller than these differences.

\section{FIVE-LOOP PREDICTIONS IN $N=1$ SUPERSYMMETRIC QCD}

We begin with the SQCD $\beta$ function in the DRED regularization scheme, where the first four coefficients are given by [8]

$$
\begin{aligned}
& \beta_{0}=3 N_{C}-N_{F}, \\
& \beta_{1}=6 N_{C}^{2}-\left[4 N_{C}-\frac{2}{N_{C}}\right] N_{F}, \\
& \beta_{2}=21 N_{C}^{3}-\left[21 N_{C}^{2}-\frac{2}{N_{C}^{2}}-9\right] N_{F}-\left[\frac{3}{N_{c}}-4 N_{C}\right] N_{F}^{2},
\end{aligned}
$$$$
\beta_{3}=A_{3}+B_{3} N_{F}+C_{3} N_{F}^{2}+C_{3} N_{F}^{2}+D_{3} N_{F}^{3},
$$

where $N_{C}$ is the number of colors, and

$$
\begin{aligned}
& A_{3}=(6+36 \alpha) N_{C}^{4}, \\
& B_{3}=-36(1+\alpha) N_{C}^{3}+(34+12 \alpha) N_{C}+\frac{8}{N_{C}}+\frac{4}{N_{C}^{3}}, \\
& C_{3}=\left(\frac{62}{3}+2 \kappa+8 \alpha\right) N_{C}^{2}-\frac{100}{3}-4 \alpha-\frac{6 \kappa-20}{3 N_{C}^{2}}, \\
& D_{3}=\frac{2}{3 N_{C}} .
\end{aligned}
$$

Here $\kappa=6 \zeta_{3}$ and $\alpha$ is a constant which has not yet been calculated exactly. Notice that there are no quartic Casimir contributions in the SQCD case. ${ }^{4}$ The APAP method was used in an earlier paper [6] to obtain the estimate $\alpha \approx 2.4$.

Proceeding now to five loops, we write

$$
\beta_{4}=A_{4}+B_{4} N_{F}+C_{4} N_{F}^{2}+D_{4} N_{F}^{3}+E_{4} N_{F}^{4} \text {. }
$$

As in the QCD case, we can input the true value of $E_{4}$ provided by a recent large- $N_{F}$ calculation [11], and given by

\footnotetext{
${ }^{4}$ Their absence may be understood as a consequence of the fact that the $\beta$ function vanishes beyond one loop for an arbitrary $N$ $=2$ supersymmetric theory. We are unable, however, to comment on the possible appearance of quartic and higher-order Casimir terms at the five-loop level.
} 
TABLE V. WAPAP's for the five-loop SQCD $\beta$ function, assuming $\alpha=2.4$. The values of $N_{F}^{\max }$ used range between 3 and 37 .

\begin{tabular}{lcccrr}
\hline \hline$N_{C}$ & 2 & 3 & 4 & 5 & \multicolumn{1}{c}{10} \\
\hline$A_{4}$ & $1.48 \times 10^{4}$ & $1.13 \times 10^{5}$ & $4.78 \times 10^{5}$ & $1.46 \times 10^{6}$ & $4.69 \times 10^{7}$ \\
$B_{4}$ & $-1.05 \times 10^{4}$ & $-5.85 \times 10^{4}$ & $-1.91 \times 10^{5}$ & $-4.72 \times 10^{5}$ & $-7.70 \times 10^{6}$ \\
$C_{4}$ & $3.25 \times 10^{3}$ & $1.29 \times 10^{4}$ & $3.21 \times 10^{4}$ & $6.42 \times 10^{4}$ & $5.29 \times 10^{5}$ \\
$D_{4}$ & -109 & -307 & -583 & -936 & $-3.87 \times 10^{3}$ \\
$E_{4}$ (input) & -3.96 & -6.64 & -9.19 & -11.7 & -23.9 \\
\hline \hline
\end{tabular}

$$
E_{4}=-\left[2 N_{C} \zeta_{3}-\left(1+2 \zeta_{3}\right) /\left(2 N_{C}\right)\right]
$$

We choose to calculate the WAPAP predictions both with and without this input. This also enables us to explore the sensitivity of the resulting prediction for $E_{4}$ to variations in $\alpha$. Assuming $\alpha=2.4$, we obtain the results shown in Table $\mathrm{V}$, whereas the results with the known values of $E_{4}$ not input are shown in Table VI. The qualitative agreement between the predicted values of $E_{4}$ in the last row of Table VI and the exact values in Table $\mathrm{V}$ is good. We note that the WAPAP process is crucial for this agreement, in that the output $E_{4}$ is quite sensitive to the value of $N_{F}^{\max }$ used, which is fixed by the WAPAP criterion. We see that the output values of $A_{4}$, $B_{4}, C_{4}$, and $D_{4}$ are quite stable, which is perhaps to be expected in view of the small numerical values of $E_{4}$. The differences between the results obtained with and without the input exact value of $E_{4}$ provide some indication of the uncertainty in the predictions. We expect, naturally, the case with input $E_{4}$ to be the more accurate.

The value $\alpha=2.4$ used above was itself based on an APAP calculation [6]. It behoves us, therefore, to explore the sensitivity of our results to the precise value of $\alpha$. In Fig. 3 we plot the WAPAP result for $E_{4}$ against $\alpha$, for $-3<\alpha<3$. We see that for this range there are two values of $\alpha$ corresponding to $E_{4}=E_{4}^{\text {exact }}$, namely, $\alpha \approx-0.9$ and $\alpha \approx 1.4$. Given the fact that in general we would expect $E_{4}$ to be the least-well-determined coefficient, we consider this result to be reasonably consistent with our previous prediction that $\alpha \approx 2.4$. It should be noted that our predictions for $A_{4}, \ldots, D_{4}$ are also sensitive to the precise value of $\alpha$.

We turn now to the alternative NSVZ prescription for the SQCD $\beta$ function, given by the following exact formula [7] which relates $\beta_{g}$ to the quark anomalous dimension $\gamma_{q}$

$$
\beta_{g}^{\mathrm{NSVZ}}=-\frac{g^{3}}{16 \pi^{2}}\left[\frac{N_{F}-3 N_{C}-2 N_{F} \gamma_{q}^{\mathrm{NSVZ}}}{1-2 N_{C} g^{2}\left(16 \pi^{2}\right)^{-1}}\right]
$$

Note the overall minus sign, in accordance with our conventions here. Using Eq. (6.5) and the result for $\gamma_{q}^{\mathrm{NSVZ}}$ given in Ref. [8], we obtain

$$
\begin{aligned}
& \beta_{0}=3 N_{C}-N_{F} \\
& \beta_{1}=6 N_{C}^{2}-\left[4 N_{C}-\frac{2}{N_{C}}\right] N_{F}, \\
& \beta_{2}=12 N_{C}^{3}-\left[12 N_{C}^{2}-\frac{2}{N_{C}^{2}}-6\right] N_{F}-\left[\frac{2}{N_{C}}-2 N_{C}\right] N_{F}^{2} \\
& \beta_{3}=A_{3}+B_{3} N_{F}+C_{3} N_{F}^{2}+D_{3} N_{F}^{3},
\end{aligned}
$$

where

$$
\begin{aligned}
& A_{3}=24 N_{C}^{4}, \\
& B_{3}=-40 N_{C}^{3}+30 N_{C}-\frac{2}{N_{C}}+\frac{4}{N_{C}^{3}}, \\
& C_{3}=(2 \kappa+14) N_{C}^{2}-24-\frac{2 \kappa-10}{N_{C}^{2}}, \\
& D_{3}=2 N_{C}-\frac{2}{N_{C}} .
\end{aligned}
$$

In this case there is no undetermined parameter $\alpha$ : we know [8] $\gamma_{q}^{\mathrm{NSVZ}}$ through three loops, and hence $\beta_{q}^{\mathrm{NSVZ}}$ through four loops.

It is possible to argue [12] on the basis of the nature of the coupling-constant redefinition connecting the two schemes that $\gamma_{q}^{\mathrm{DRED}}$ and $\gamma_{q}^{\mathrm{NSVZ}}$ are the same at leading order in $N_{F}$. Hence, if as before we write

$$
\beta_{4}=A_{4}+B_{4} N_{F}+C_{4} N_{F}^{2}+D_{4} N_{F}^{3}+E_{4} N_{F}^{4},
$$

TABLE VI. WAPAP's for the five-loop SQCD $\beta$ function, again assuming $\alpha=2.4$, but without the exact values of $E_{4}$ as input. The values of $N_{F}^{\max }$ used range between 4 and 61 .

\begin{tabular}{lcccrr}
\hline \hline$N_{C}$ & 2 & 3 & 4 & 5 & \multicolumn{1}{c}{10} \\
\hline$A_{4}$ & $1.46 \times 10^{4}$ & $1.12 \times 10^{5}$ & $4.73 \times 10^{5}$ & $1.45 \times 10^{6}$ & $4.64 \times 10^{7}$ \\
$B_{4}$ & $-1.04 \times 10^{4}$ & $-5.87 \times 10^{4}$ & $-1.91 \times 10^{5}$ & $-4.74 \times 10^{5}$ & $-7.73 \times 10^{6}$ \\
$C_{4}$ & $3.16 \times 10^{3}$ & $1.25 \times 10^{4}$ & $3.11 \times 10^{4}$ & $6.21 \times 10^{4}$ & $5.12 \times 10^{5}$ \\
$D_{4}$ & -134 & -400 & -767 & $-1.24 \times 10^{3}$ & $-5.12 \times 10^{3}$ \\
$E_{4}$ & -2.44 & -4.53 & -6.33 & -8.03 & -16.1 \\
\hline \hline
\end{tabular}




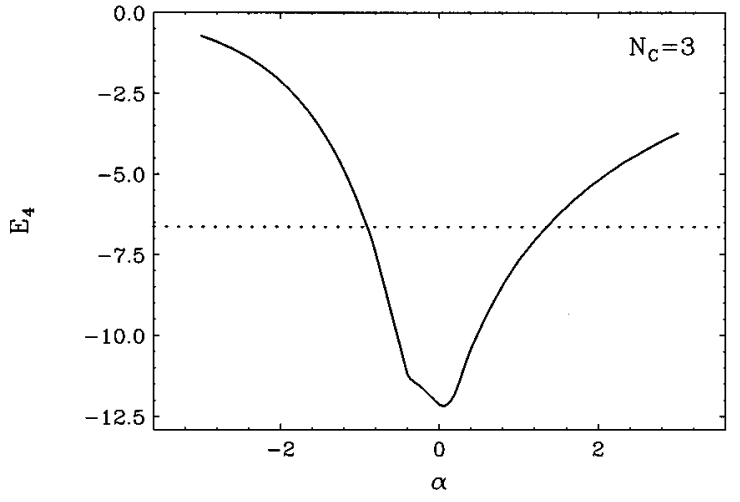

FIG. 3. The WAPAP result for $E_{4}$ plotted against $\alpha$, for $-3<\alpha<3$.

we can find $E_{4}$, as we did in the DRED case, from the large$N_{F}$ results in Ref. [11]. The result is

$$
E_{4}=2[1-2 \zeta(3)]\left(N_{C}-1 / N_{C}\right) .
$$

We also have, as is evident from Eq. (6.5), that $A_{4}=48 N_{C}^{5}$, providing an additional check on our calculation. ${ }^{5}$ Our WAPAP results are shown in the Tables VII and VIII, for the cases with and without $E_{4}$ input. Also shown in the second row of Table VIII are the exact results for $A_{4}$.

We see that the WAPAP's are in general in good agreement with the exact result for $A_{4}$ in the NSVZ scheme, at the $10 \%$ level. Although encouraging, these results are not quite as compelling as the ones for the DRED scheme. This is at first sight surprising, given the form of Eq. (6.5), which appears at first sight to be close to the rational function form of the PA's. However, as mentioned in Sec. I, perhaps minimal subtraction schemes are more amenable to Padé techniques. The anomalously poor result for $A_{4}$ in Table VII is caused by the fact that the error $\delta_{4}$ is close to -1 in this case, for the $N_{F}^{\max }$ values corresponding to the determination of $D_{4}$. The reason the result for $D_{4}$ is not also anomalously large is that the two values from which the weighted average is taken are both numerically large but with opposite signs. Thus we cannot rely on either the $A_{4}$ or $D_{4}$ prediction for $N_{C}=5$. With this exception, $A_{4}$ comes out reasonably close to the exact result. This means, of course that the predictions for $B_{4}, \ldots, D_{4}$ will not change much if we input $A_{4}$ as well as

\footnotetext{
${ }^{5} \mathrm{We}$ could, of course, input both $A_{4}$ and $E_{4}$, but we choose instead to compare the WAPAP results for all the five-loop coefficients with the corresponding ones with $E_{4}$ input.
}

$E_{4}$. Analogously to the five-loop QCD case discussed in Sec. V, we take the differences between the entries in Tables VII and VIII as lower limits on the possible uncertainties in our five-loop NSVZ predictions.

\section{QUARK MASS ANOMALOUS DIMENSION IN QCD}

We now consider the quark mass anomalous dimension $\gamma$ in QCD, defined as

$$
\gamma=\frac{d \ln m_{q}}{d \ln \mu^{2}}=-\gamma_{0} a-\gamma_{1} a^{2}-\gamma_{2} a^{3}-\gamma_{3} a^{4}-\gamma_{4} a^{5}+O\left(a^{6}\right),
$$

where $a=\alpha_{s} / \pi$. The four-loop coefficient $\gamma_{3}$ was recently computed in Refs. $[13,14]$, and the full exact results for the coefficients $\gamma_{n}$ for $n=0,1,2$, and 3 are given by

$$
\begin{aligned}
& \gamma_{0}=\frac{1}{4}\left[3 C_{F}\right], \\
& \gamma_{1}=\frac{1}{16}\left[\frac{3}{2} C_{F}^{2}+\frac{97}{6} C_{F} C_{A}-\frac{10}{3} C_{F} T_{F} N_{F}\right] \\
& \gamma_{2}=\frac{1}{64}\left[\frac{129}{2} C_{F}^{3}-\frac{129}{4} C_{F}^{2} C_{A}+\frac{11413}{108} C_{F} C_{A}^{2}+C_{F}^{2} T_{F} N_{F}(-46\right. \\
& \left.\left.+48 \zeta_{3}\right)+C_{F} C_{A} T_{F} N_{F}\left(-\frac{556}{27}-48 \zeta_{3}\right)-\frac{140}{27} C_{F} T_{F}^{2} N_{F}^{2}\right], \\
& \gamma_{3}=\frac{1}{256}\left[C_{F}^{4}\left(-\frac{1261}{8}-336 \zeta_{3}\right)+C_{F}^{3} C_{A}\left(\frac{15349}{12}+316 \zeta_{3}\right)\right. \\
& +C_{F}^{2} C_{A}^{2}\left(-\frac{34045}{36}-152 \zeta_{3}+440 \zeta_{5}\right)+C_{F} C_{A}^{3}\left(\frac{70055}{72}\right. \\
& \left.+\frac{1418}{9} \zeta_{3}-440 \zeta_{5}\right)+C_{F}^{3} T_{F} N_{F}\left(-\frac{280}{3}+552 \zeta_{3}-480 \zeta_{5}\right) \\
& +C_{F}^{2} C_{A} T_{F} N_{F}\left(-\frac{8819}{27}+368 \zeta_{3}-264 \zeta_{4}+80 \zeta_{5}\right) \\
& +C_{F} C_{A}^{2} T_{F} N_{F}\left(-\frac{65459}{162}-\frac{2684}{3} \zeta_{3}+264 \zeta_{4}+400 \zeta_{5}\right) \\
& +C_{F}^{2} T_{F}^{2} N_{F}^{2}\left(\frac{304}{27}-160 \zeta_{3}+96 \zeta_{4}\right)+C_{F} C_{A} T_{F}^{2} N_{F}^{2}\left(\frac{1342}{81}\right. \\
& \left.+160 \zeta_{3}-96 \zeta_{4}\right)+C_{F} T_{F}^{3} N_{F}^{3}\left(-\frac{664}{81}+\frac{128}{9} \zeta_{3}\right) \\
& +\frac{d_{F}^{a b c d} d_{A}^{a b c d}}{d_{Q}}\left(-32+240 \zeta_{3}\right)+N_{F} \frac{d_{F}^{a b c d} d_{F}^{a b c d}}{d_{Q}}(64 \\
& \left.\left.-480 \zeta_{3}\right)\right]
\end{aligned}
$$

where for $S U\left(N_{C}\right)$ the quadratic and quartic Casimirs are as defined in Eqs. (3.2) and (3.5), and $T_{F}=\frac{1}{2}$ as before. In addition, $d_{Q}$ is the dimension of the quark representation, so

TABLE VII. WAPAP's for the five-loop NSVZ $\beta$ function, with the exact values of $E_{4}$ used as input. The values of $N_{F}^{\max }$ used range between 3 and 26 .

\begin{tabular}{lccccc}
\hline \hline$N_{C}$ & 2 & 3 & 4 & 5 & \multicolumn{1}{c}{10} \\
\hline$A_{4}$ & $1.68 \times 10^{3}$ & $1.04 \times 10^{4}$ & $4.44 \times 10^{4}$ & $4.99 \times 10^{5}$ & $4.42 \times 10^{6}$ \\
$B_{4}$ & $-1.25 \times 10^{3}$ & $-7.87 \times 10^{3}$ & $-2.63 \times 10^{4}$ & $-6.56 \times 10^{4}$ & $-1.08 \times 10^{6}$ \\
$C_{4}$ & 750 & $3.11 \times 10^{3}$ & $7.87 \times 10^{3}$ & $1.58 \times 10^{4}$ & $1.32 \times 10^{5}$ \\
$D_{4}$ & -6.0 & -90.1 & -163 & -516 & -938 \\
$E_{4}$ (input) & -4.21 & -7.49 & -10.5 & -13.5 & -27.8 \\
\hline \hline
\end{tabular}


TABLE VIII. WAPAP's for the five-loop NSVZ $\beta$ function, with $E_{4}$ not input. The values of $N_{F}^{\max }$ used range between 4 and 25 .

\begin{tabular}{lccccc}
\hline \hline$N_{C}$ & 2 & 3 & 4 & 5 & 10 \\
\hline$A_{4}$ & $1.49 \times 10^{3}$ & $1.05 \times 10^{4}$ & $4.33 \times 10^{4}$ & $1.42 \times 10^{5}$ & $4.45 \times 10^{6}$ \\
$A_{4}$ (exact) & $1.536 \times 10^{3}$ & $1.166 \times 10^{4}$ & $4.915 \times 10^{4}$ & $1.500 \times 10^{5}$ & $4.800 \times 10^{6}$ \\
$B_{4}$ & $-1.13 \times 10^{3}$ & $-7.80 \times 10^{3}$ & $-2.65 \times 10^{4}$ & $-6.64 \times 10^{4}$ & $-1.09 \times 10^{6}$ \\
$C_{4}$ & 612 & $2.87 \times 10^{3}$ & $7.35 \times 10^{3}$ & $1.48 \times 10^{4}$ & $1.23 \times 10^{5}$ \\
$D_{4}$ & -75.2 & -241 & -462 & -742 & -3060 \\
$E_{4}$ & -13.0 & -13.3 & -15.8 & -27.9 & -50.6 \\
\hline \hline
\end{tabular}

that $d_{Q}=N_{C}$ for $S U\left(N_{C}\right)$, and we have $\zeta_{4} \equiv \zeta(4)$ $=1.0823232 \ldots$ and $\zeta_{5} \equiv \zeta(5)=1.0369277 \ldots$. For $N_{C}=3$, we have

$\gamma_{0}=1$,

$\gamma_{1}=\frac{1}{16}\left[\frac{202}{3}-\frac{20}{9} N_{F}\right]$

$\gamma_{2}=\frac{1}{64}\left[1249+\left(-\frac{2216}{27}-\frac{160}{3} \zeta_{3}\right) N_{F}-\frac{140}{81} N_{F}^{2}\right]$,

$\gamma_{3}=\frac{1}{256}\left[\frac{4603055}{162}+\frac{135680}{27} \zeta_{3}-8800 \zeta_{5}+\left(-\frac{91723}{27}-\frac{34192}{9} \zeta_{3}\right.\right.$

$\left.+880 \zeta_{4}+\frac{18400}{9} \zeta_{5}\right) N_{F}+\left(\frac{5242}{243}+\frac{800}{9} \zeta_{3}-\frac{160}{3} \zeta_{4}\right) N_{F}^{2}$

$\left.+\left(-\frac{332}{243}+\frac{64}{27} \zeta_{3}\right) N_{F}^{3}\right]$

which have the numerical values

$\gamma_{0}=1$,

$\gamma_{1} \approx 4.20833-0.138889 N_{F}$,

$\gamma_{2} \approx 19.5156-2.28412 N_{F}-0.0270062 N_{F}^{2}$,

$\gamma_{3} \approx 98.9434-19.1075 N_{F}+0.276163 N_{F}^{2}+0.00579322 N_{F}^{3}$.
Omitting the quartic Casimir contributions, one obtains $\gamma_{3}=96.4386-18.8292 N_{F}+0.276163 N_{F}^{2}+0.00579322 N_{F}^{3}$,

and we shall now compare Eqs. (7.4) and (7.5) with APAP's.

It transpires that the WAPAP procedure does not work so well here. The most accurate results for both $B_{3}^{\gamma}$ and $C_{3}^{\gamma}$ are obtained for small $N_{F}^{\max }$. This is reasonably consistent with the WAPAP behavior in the $C_{3}^{\gamma}$ case: here, the weight difference $C_{3}^{\gamma W}-A_{3}^{\gamma W}$ never changes sign, but is smallest at $N_{F}^{\max }=2$ on the edge of the range. However, the WAPAP criterion for $B_{3}^{\gamma}$ leads to values of $N_{F}^{\max }$ which start at 9 for $N_{C}=2$ and increase with $N_{C}$. Nevertheless, as in the previous sections, it seems sensible to match at negative $N_{F}$, and spectacular results are obtained if we simply take $N_{F}^{\max }=4$ (with $-N_{F}^{\max }<N_{F}<0$ ) throughout, as can be seen from Table IX, where numerical predictions for the coefficients in the parametrization,

$$
\gamma_{3}=A_{3}^{\gamma}+B_{3}^{\gamma} N_{F}+C_{3}^{\gamma} N_{F}^{2}+D_{3}^{\gamma} N_{F}^{3},
$$

are given both without (without $Q$ ) and with (with $Q$ ) quartic Casimir contributions. It should be noted that we have used as input the exact result for $D_{3}^{\gamma}$, which is contained in Eq. (7.2).

TABLE IX. Four-loop quark mass anomalous dimension in QCD: APAP's for fixed $N_{F}^{\max }=4$ are compared with the exact values both without and with $Q$, the quartic Casimir terms.

\begin{tabular}{|c|c|c|c|c|c|}
\hline & \multicolumn{5}{|c|}{$N_{C}$} \\
\hline & 2 & 3 & 4 & 5 & 20 \\
\hline \multicolumn{6}{|c|}{$A_{3}^{\gamma}$} \\
\hline APAP & 16.1 & 97.9 & 328 & 822 & $2.18 \times 10^{5}$ \\
\hline without $Q$ & 15.4 & 96.4 & 327 & 825 & $2.23 \times 10^{5}$ \\
\hline with $Q$ & 16.0 & 98.9 & 334 & 840 & $2.26 \times 10^{5}$ \\
\hline \multicolumn{6}{|c|}{$B_{3}^{\gamma}$} \\
\hline APAP & -5.14 & -20.0 & -49.3 & -98.0 & $-6.39 \times 10^{3}$ \\
\hline without $Q$ & -4.70 & -18.8 & -47.1 & -94.2 & $-6.27 \times 10^{3}$ \\
\hline with $Q$ & -4.77 & -19.1 & -48.0 & -96.2 & $-6.43 \times 10^{3}$ \\
\hline \multicolumn{6}{|c|}{$C_{3}^{\gamma}$} \\
\hline APAP & 0.065 & 0.224 & 0.478 & 0.828 & 17.5 \\
\hline exact & 0.111 & 0.276 & 0.504 & 0.796 & 13.0 \\
\hline \multicolumn{6}{|c|}{$D_{3}^{\gamma}$} \\
\hline input & $3.26 \times 10^{-3}$ & $5.79 \times 10^{-3}$ & $8.15 \times 10^{-3}$ & 0.0104 & 0.0433 \\
\hline
\end{tabular}


TABLE X. APAP's for the five-loop quark mass anomalous dimension in QCD, calculated with and without the four-loop quartic Casimir terms.

\begin{tabular}{lccccc}
\hline \hline$N_{C}$ & 2 & 3 & 4 & 5 & 20 \\
\hline$A_{4}($ with $Q)$ & 56.0 & 530 & $2.41 \times 10^{3}$ & $7.63 \times 10^{3}$ & $8.37 \times 10^{6}$ \\
$A_{4}($ without $Q)$ & 50.5 & 493 & $2.27 \times 10^{3}$ & $7.22 \times 10^{3}$ & $7.97 \times 10^{6}$ \\
$B_{4}($ with $Q)$ & -23.3 & -143 & -483 & $-1.22 \times 10^{3}$ & $-3.33 \times 10^{5}$ \\
$B_{4}($ without $Q)$ & -21.7 & -135 & -457 & $-1.15 \times 10^{3}$ & $-3.12 \times 10^{5}$ \\
$C_{4}($ with $Q)$ & 1.70 & 6.67 & 16.8 & 33.7 & $2.29 \times 10^{3}$ \\
$C_{4}($ without $Q)$ & 1.64 & 6.44 & 16.0 & 32.0 & $2.14 \times 10^{3}$ \\
$D_{4}($ with $Q)$ & $8.12 \times 10^{-3}$ & 0.037 & 0.0891 & 0.165 & 4.31 \\
$D_{4}($ without $Q)$ & $8.88 \times 10^{-3}$ & 0.037 & 0.0831 & 0.148 & 3.48 \\
$E_{4}$ (input) & $-4.80 \times 10^{-5}$ & $-8.54 \times 10^{-5}$ & $-1.2 \times 10^{-4}$ & $-1.54 \times 10^{-4}$ & $-6.39 \times 10^{-4}$ \\
\hline \hline
\end{tabular}

It can be seen that in all cases the APAP estimate is quite accurate over a wide range of $N_{C}$. In most cases, the APAP estimate is closer to the exact result without the quartic Casimir contribution (without $Q$ ), but in any case the quartic Casimir contribution to $\gamma_{3}$ is smaller than in the case of the QCD $\beta$ function.

We now go on to discuss the five-loop APAP estimate of $\gamma$. We parametrize the five-loop quark mass anomalous dimension $\gamma_{4}$ in the form

$$
\gamma_{4}=A_{4}^{\gamma}+B_{4}^{\gamma} N_{F}+C_{4}^{\gamma} N_{F}^{2}+D_{4}^{\gamma} N_{F}^{3}+E_{4}^{\gamma} N_{F}^{4},
$$

where the value of $E_{4}^{\gamma}$ can be derived from [15]

$$
E_{4}^{\gamma}=C_{F} T_{F}^{4}\left(-65 / 5184-5 \zeta(3) / 324+\pi^{4} / 3240\right) \text {. }
$$

We use the full $\gamma_{3}$ as input, including the quartic Casimir contribution. As we argued in the case of the QCD $\beta$ function, we expect our five-loop estimate to include the effects of contributions involving such quartic Casimir terms, but not the effect of new Casimir terms making a first appearance. Once again we choose $N_{F}^{\max }=4$ to derive the results shown in Table X.

\section{ABELIAN GAUGE THEORIES}

All of the previous sections have dealt with APAP predictions for non-Abelian theories. It is natural to ask whether similarly accurate results can be obtained for the Abelian case. We address this question in this section, choosing as our example the fermion mass anomalous dimension with $N_{F}$ charged fermions, where good results were found in the non-Abelian case, as we saw in Sec. VII. A supplementary reason for choosing this example is that the next-to-leading$N_{F}$ result is available, as well as the leading one.

The results for $\gamma_{1}, \ldots, \gamma_{3}$ in the Abelian case follow from Eq. (7.2) by setting

$$
C_{F}=T_{F}=1, \quad C_{A}=0, \quad \frac{d_{F}^{a b c d} d_{A}^{a b c d}}{d_{Q}}=0, \quad \frac{d_{F}^{a b c d} d_{F}^{a b c d}}{d_{Q}}=1,
$$

so that

$$
\begin{gathered}
\gamma_{0}=0.75, \\
\gamma_{1} \approx 0.09375-0.2083 N_{F},
\end{gathered}
$$

$$
\begin{gathered}
\gamma_{2} \approx 1.0078+0.18279 N_{F}-0.08102 N_{F}^{2}, \\
\gamma_{3} \approx-2.1934-1.7207 N_{F}-0.30143 N_{F}^{2}+0.03476 N_{F}^{3} .
\end{gathered}
$$

Omitting the quartic Casimir term, we would instead have

$$
\gamma_{3} \approx-2.1934+0.2831 N_{F}-0.30143 N_{F}^{2}+0.03476 N_{F}^{3} .
$$

We can see at once that the miraculous success of the previous APAP prediction for $\gamma_{3}$ will not be reproduced here. For $N_{F}=0$, the quenched case, the sign of $\gamma_{3}$ differs from the sign of $\gamma_{2}^{2} / \gamma_{1}$. Moreover, $\gamma_{1}$ has a zero, and hence $\gamma_{3}^{\text {APAP }}$ has a pole, for $N_{F} \approx 0.45$. Hence, we cannot hope to reproduce $\gamma_{3}$ for small values of $\left|N_{F}\right|$. For large $\left|N_{F}\right|$ the sign of $\gamma_{3}$ is still wrong, so the method fails in this region also.

One easily verifies that this pessimism is confirmed by the results, and things do not improve at five loops. Then, as well as $E_{4}^{\gamma}$ as given in Eq. (7.8), it is possible to derive from [16] the result for $D_{4}$ :

$$
D_{4}^{\gamma}=\frac{11}{96} \zeta_{3}+\frac{1}{6} \zeta_{5}-\frac{\pi^{4}}{288}+\frac{4483}{41472} \approx 0.0804 .
$$

We notice now, however, that $\gamma_{2}$ has zeros, and hence $\gamma_{3}^{\text {APAP }}$ has poles, for $N_{F}=-2.6$ and 4.8. Consequently, we may expect that the results will be rather sensitive to the range of $N_{F}$, if we match in a region including the origin. Of course, in the Abelian theory we cannot expect smooth behavior as we pass through $N_{F}=0$-perhaps the occurrence of poles near to $N_{F}=0$ on both sides is simply a confirmation of this? On the other hand, for large $N_{F}$ we have $\gamma_{3}^{2} / \gamma_{2} \approx$ $-0.014 N_{F}^{4}$, whereas $E_{4}^{\gamma} \approx-0.001$, so we also cannot expect good results at increasing $\left|N_{F}\right|$.

We leave it to the reader to convince her(him)self that we cannot expect to extract reliable predictions for $A_{4}, \ldots, C_{4}$. We also record that the QED and SQED gauge $\beta$ functions yield similarly unattractive results. Evidently, Abelian theories are less amenable to the APAP approach, for some unknown reason.

\section{CONCLUSIONS}

We have presented results obtained from our APAP method for the four-loop and five-loop QCD $\beta$-function co- 
efficients, for the five-loop SQCD $\beta$-function coefficients, and for the four- and five-loop quark mass anomalous dimensions in QCD. Particularly in the case of the QCD $\beta$ function, and to some extent also for SQCD, particularly in the DRED scheme, a modified procedure for extracting the predictions for the various coefficients of powers of $N_{F}$ (WAPAP) gave improved results. In general, the four-loop results agree very well with the known results, giving us confidence in our predictions of the five-loop terms.

Our four-loop QCD $\beta$-function predictions [5] were confirmed very rapidly by an exact calculation [9]. Unfortunately, in view of the current limitations on the technology of exact perturbative calculations in QCD and SQCD, it may be some time before our five-loop predictions can also be tested directly. It would therefore be interesting to find alternative techniques that could be confronted or combined with APAP's. One possible complementary technique may be that of the large- $N_{F}$ expansion. Unfortunately, it is the leading term in $N_{F}$ which is least well determined by the APAP approach, which is related to the poor results obtained in the Abelian case. It would be very interesting if the large- $N_{F}$ methods could be extended to next-to-leading terms in this expansion for the non-Abelian case, in which case more comparisons and cross-checks could be made.

\section{ACKNOWLEDGMENTS}

This work was supported by the U.S. Department of Energy under Contract No. DE-AC03-765F00515 and Grant No. DE-FG05-84ER40215. The research of M.K. was supported in part by the Israel Science Foundation administered by the Israel Academy of Sciences and Humanities, and by a Grant from the G.I.F., the German-Israeli Foundation for Scientific Research and Development. I.J. and D.R.T.J. thank John Gracey for help with his large- $N_{F}$ results.
[1] For recent reviews and references, see M. Beneke, talk at the Fifth International Workshop on Deep Inelastic Scattering and QCD, Chicago 1997, hep-ph/9706457; R. Akhoury and V. I. Zakharov, in $Q C D$ 96, Proceedings of the Conference, France, 1996, edited by S. Narison [Nucl. Phys. B (Proc. Suppl.) 54A, (1997)]; hep-ph/9710257, Yu. L. Dokshitser and B. R. Webber, Phys. Lett. B 404, 321 (1997).

[2] See, for example, T. van Ritbergen, J. A. M. Vermaseren, S. A. Larin, and P. Nogueira, Int. J. Mod. Phys. C 6, 513 (1995); A. L. Kataev, talk at Second Workshop on Continuous Advances in QCD, Minneapolis, 1996, hep-ph/9607426; see also Refs. [9], [13], and [14].

[3] M. A. Samuel, G. Li, and E. Steinfelds, Phys. Rev. D 48, 869 (1993); Phys. Lett. B 323, 188 (1994); M. A. Samuel and G. Li, Int. J. Theor. Phys. 33, 1461 (1994); Phys. Lett. B 331, 114 (1994).

[4] M. A. Samuel, J. Ellis, and M. Karliner, Phys. Rev. Lett. 74, 4380 (1995); J. Ellis, E. Gardi, M. Karliner, and M. A. Samuel, Phys. Lett. B 366, 268 (1996); Phys. Rev. D 54, 6986 (1996); E. Gardi, ibid. 56, 68 (1997); S. J. Brodsky, J. Ellis, E. Gardi, M. Karliner, and M. A. Samuel, Phys. Rev. D 56, 6980 (1997). [5] J. Ellis, M. Karliner, and M. A. Samuel, Phys. Lett. B 400, 176
(1997).

[6] I. Jack, D. R. T. Jones, and M. A. Samuel, Phys. Lett. B 407, 143 (1997).

[7] V. Novikov et al. Nucl. Phys. B229, 381 (1983); Phys. Lett. 166B, 329 (1986); M. Shifman and A. Vainshtein, Nucl. Phys. B277, 456 (1986); A. Vainshtein, V. Zakharov, and M. Shifman, Sov. J. Nucl. Phys. 43, 1028 (1986); M. Shifman, A. Vainshtein, and V. Zakharov, Phys. Lett. 166B, 334 (1986).

[8] I. Jack, D. R. T. Jones, and C. G. North, Nucl. Phys. B486, 479 (1997).

[9] T. van Ritbergen, J. A. M. Vermaseren, and S. A. Larin, Phys. Lett. B 400, 379 (1997).

[10] J. A. Gracey, Phys. Lett. B 373, 178 (1996).

[11] P. M. Ferreira, I. Jack, D. R. T. Jones, and C. G. North, Nucl. Phys. B504, 108 (1997).

[12] I. Jack and D. R. T. Jones, hep-ph/9707278.

[13] J. A. M. Vermaseren, S. A. Larin, and T. van Ritbergen, Phys. Lett. B 405, 327 (1997).

[14] K. G. Chetyrkin, Phys. Lett. B 404, 161 (1997).

[15] A. Palanques-Mestre and P. Pascual, Commun. Math. Phys. 95, 277 (1984).

[16] J. A. Gracey, Phys. Lett. B 317, 415 (1993). 